\title{
Um medidor de luminosidade com módulo sensor integrado e aquisição automática de dados com aplicações didáticas
}

\author{
A light meter with integrated sensor module and automatic data acquisition with didactic applications \\ Paulo H. Guadagnini*1] ${ }^{*}$ Fábio Saraiva da Rocha ${ }^{2}$, Vania E. Barlette ${ }^{1}$ \\ ${ }^{1}$ Universidade Federal do Pampa, Campus Bagé, RS, Brasil \\ ${ }^{2}$ Universidade Federal de Pelotas, Pelotas, RS, Brasil
}

Recebido em 22 de Outubro, 2018. Revisado em 06 de Dezembro, 2018. Aceito em 15 de Dezembro, 2018.

\begin{abstract}
Este trabalho apresenta o projeto de um medidor de luz para atividades didáticas experimentais utilizando o sensor de luz integrado BH1750. O medidor de luz é construído conectando o sensor BH1750 com a plataforma Arduino. Os dados são transferidos para um computador e são visualizados durante a execução de um experimento por meio de tabelas e gráficos em tempo real. São apresentadas as características físicas e operacionais do sensor BH1750, bem como o projeto eletrônico e a programação computacional. Os resultados de desempenho do medidor de luz proposto neste trabalho em comparação a um de referência indicam que não há diferença estatisticamente significante entre eles em relação às medidas de iluminância. Para ilustrar alguns usos didáticos do medidor de luz desenvolvido neste trabalho, é apresentada uma investigação da dependência da atenuação de luz com a distância, assim como um fotômetro de LED para estudos em espectroscopia de absorção molecular e uma investigação da variação de iluminância da lâmpada fluorescente. Ressalta-se, ainda, o potencial de uso de um medidor de luz como o apresentado aqui nas práticas de ensino de ciências que buscam a inovação e o engajamento dos alunos em projetos interdisciplinares.
\end{abstract}

Palavras-chave: Ensino de ciências, Sensor de luminosidade, Aquisição automática de dados, fotômetro de LED.

This work presents the design of a light meter for experimental didactic activities using the integrated light sensor BH1750. The light meter is built by connecting the BH1750 sensor with the Arduino platform. The data are transferred to a computer and they are viewed during the execution of an experiment by means of tables and graphs in real time. The physical and operational characteristics of the BH1750 sensor are presented, as well as the electronic design and computational programming. Results of performance of the light meter proposed in this work compared to a reference one indicates there is no statistically significant difference between them concerning the illuminance measurements. For illustrating didactic some uses of the light meter developed in this work, an investigation of the dependence of light attenuation with distance is presented, as well as a LED photometer for studies in molecular absorption spectroscopy and an investigation of the fluorescent lamp illuminance variation. It is also emphasized the potential use of a light meter like that presented here in science teaching practices seeking innovation and the engagement of students in interdisciplinary projects.

Keywords: Science teaching, Light meter, Automatic data acquisition, LED photometer.

\section{Introdução}

Medidas de luminosidade são importantes em estudos envolvendo emissão ou absorção de radiação luminosa, por exemplo, em avaliações de eficiência de fontes luminosas, luminosidade de ambientes e investigações que envolvem interação da radiação com a matéria. Nessa linha de aplicações, este trabalho descreve o projeto e construção de um medidor de luminosidade que inclui a possibilidade de aquisição automática de dados, podendo ser aplicado em atividades práticas experimentais no ensino de ciências, tais como investigações sobre atenuação da radiação em função da distância, absorção de radiação luminosa por

*Endereço de correspondência: pguadagnini@gmail.com solutos em soluções, e avaliação de lâmpadas quanto a variação de sua intensidade luminosa no tempo.

Atualmente há no mercado diversos instrumentos comerciais para medidas de luminosidade projetados para aplicações práticas experimentais que, em geral, envolvem um alto custo financeiro para a nossa realidade educacional. Esses instrumentos didáticos são projetados, na maioria dos casos, a partir de componentes eletrônicos e módulos sensores de baixo custo financeiro, baseados em princípios físicos conhecidos, porém possuem elevado valor agregado. Os projetos dessa categoria de equipamentos são protegidos por propriedade industrial, o que dificulta a compreensão detalhada do seu modo de funcionamento e restringe as possibilidades de uso de softwares de aquisição de dados não proprietários. Uma alternativa, explorada neste trabalho, é o uso de projetos abertos ba- 
seados em módulos sensores de baixo custo e plataformas microcontroladas abertas.

O mercado internacional tem disponibilizado módulos sensores que, se interligados a uma eletrônica adequada, podem medir diversas grandezas físicas. Esses módulos sensores podem ser interligados a dispositivos microcontroladores (também popularizados e vendidos a baixo custo) que executam processamento de dados, digitalização de sinais analógicos, envio e recebimento de dados e comandos de um computador. O projeto aqui apresentado utiliza o módulo sensor de luz integrado BH1750 de baixo custo e a plataforma microcontrolada Arduino, juntamente com o software Excel for Arduino para aquisição automática de dados na planilha eletrônica Excel. São apresentadas as características físicas do sensor, a constituição básica do projeto, desde componentes eletrônicos até a programação computacional envolvida em todo o processo. Apontamos possibilidades de uso em práticas experimentais e uma aplicação na construção de um fotômetro de LED simples.

Embora já exista uma comunidade de pesquisadores voltada ao desenvolvimento de projetos de instrumentação eletrônica de baixo custo, baseados em módulos sensores para uso em laboratórios didáticos de ensino de ciências [1-4], o número de projetos sobre sistemas automáticos de aquisição e tratamento de dados assistidos por computador ainda é pequeno para estimular comunidades da educação básica e superior a reproduzirem tais projetos e/ou construírem seus próprios equipamentos. Projetos abertos com módulos sensores que utilizam aquisição automática de dados tem a potencialidade de promover acessibilidade às tecnologias atuais e suprir parte da demanda de renovação do instrumental de coleta de dados experimentais para professores e escolas que não contam com grandes recursos para a compra de modernos equipamentos comerciais.

Tais recursos têm representado inovação nas formas de ensinar e aprender ainda pouco difundidas. As experiências já acumuladas em diversos trabalhos [5-11] sugerem vários benefícios de se utilizar aquisição automática de dados no ensino de ciências, tais como o enriquecimento das experiências de aprendizagem, a possibilidade de se efetuar experimentos cuja coleta de dados é feita em intervalos de tempo muito pequeno (frações de segundo) ou muito longos (horas), agilidade na coleta dos dados com mais tempo para análise dos dados e interpretação do fenômeno em estudo, alfabetização científica e tecnológica que decorre da experiência prática de se utilizar sistemas de aquisição automática de dados, e o uso da argumentação em atividades práticas experimentais.

Uma característica comum nesses estudos é a possibilidade de abordagens investigativas dos fenômenos em estudo, em que os estudantes podem fazer prognósticos e esboços do comportamento das grandezas de interesse, discutir com os demais colegas do grupo e estabelecer suas próprias conclusões durante a dinâmica de uma experiência. Em termos gerais, atividades investigati- vas são planejadas para que os estudantes, na interação com seus pares, em pequenos grupos, apreendam estratégias de pensamento científico que envolvem mobilizar conhecimentos teóricos na análise e interpretação de uma situação de interesse, e testar suas explicações numa perspectiva investigativa.

As atividades, nessa perspectiva, podem envolver um ciclo de aprendizagem que envolve desde o levantamento de hipóteses para a solução ou compreensão de um problema de interesse, o teste de hipóteses por meio do planejamento e execução de experimentos, e o tratamento dos resultados experimentais obtidos (em geral, representados sob a forma de tabelas e gráficos gerados utilizando sensores e sistemas de aquisição de dados), até a interpretação dos resultados e a conclusão do experimento, finalizando com o confronto entre a conclusão experimental e as hipóteses feitas. Esse ciclo de aprendizagem possibilita ao estudante relacionar hipóteses com conclusões experimentais de maneira fundamentada. Enfatiza-se, no entanto, que o caráter investigativo está na didática utilizada, e que os recursos tecnológicos contribuem para operacionalizar a atividade.

O plano do artigo consiste em apresentar na seção III os princípios físicos do funcionamento de um sensor de luminosidade semicondutor e, na seção seguinte, apresentar o projeto de construção do sensor de luminosidade e testes de desempenho comparando-se com um medidor comercial de referência. No sentido de fornecer algumas ideias de aplicações didáticas do sensor de luminosidade, a seção IV apresenta aplicações práticas de interesse no ensino de física e no ensino de química.

\section{Aspectos físicos de um sensor de luminosidade}

A fotocondutividade está baseada no efeito fotoelétrico descrito por A. Einstein e é um fenômeno quântico associado à variação de condutividade elétrica devido a interação de fótons da radiação eletromagnética excitando elétrons e gerando lacunas na rede de íons que compõe um material. Em um semicondutor, por exemplo, a energia entregue pelos fótons pode promover um acréscimo na densidade de elétrons na banda de condução do material favorecendo a condutividade e alterando a resistividade elétrica. A Fig. 1 apresenta o diagrama de bandas referente ao processo de excitação de portadores da banda de valência para a banda de condução vencendo o gap de energia proibida após a interação com os fótons. Neste caso a energia do fóton de excitação deve ser maior que o gap.

A estrutura mais simples de um fotodiodo, usado no modo fotocondutivo sobre o qual se baseiam os detectores de luminosidade, consiste de uma junção semicondutora $\mathrm{p}$ (lacunas) - n (elétrons). A Fig. 1 mostra o fotodiodo exposto à radiação de frequência específica e acoplado a uma fonte de tensão polarizando reversamente o dispositivo. A junção semicondutora do diodo apresenta uma 


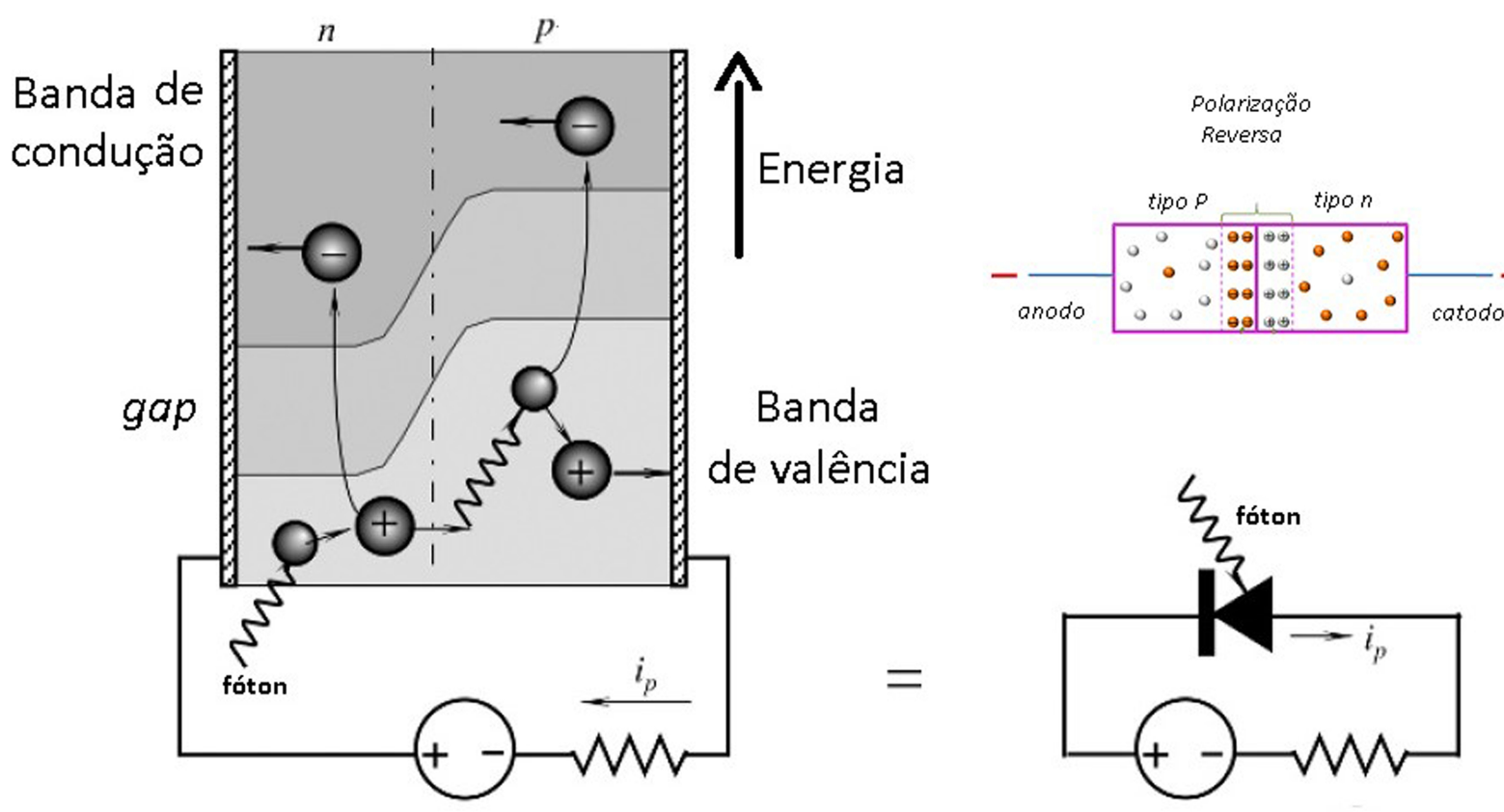

Figura 1: Estrutura básica de um fotodiodo em polarização reversa (reverse bias mode). Adaptado da Ref. [12].

zona de transição (ou depleção) que é caracterizada pela existência, em seu interior, de um forte campo elétrico gerado por cargas elétricas fixas na matriz iônica originadas pela mobilidade de portadores livres. Durante a formação da junção, os portadores livres migram para o lado reverso. Após cessar a mobilidade de cargas, se estabelece o equilíbrio com uma barreira de potencial elétrico que barra a migração de portadores através da junção.

No modo de polarização reversa do diodo (Fig. 1) é aplicada uma tensão positiva ao cátodo e negativa ao ânodo. A polarização tem a mesma direção que a do campo elétrico da região de transição. Isso aumentará a força aplicada aos portadores de carga ativados pelos fótons incidentes gerando uma fotocorrente elétrica $I p$. O potencial positivo aplicado ao cátodo atrairá buracos para a região da junção e o potencial negativo no ânodo atrairá elétrons para o lado cátodo da junção. Isso faz com que a zona de transição aumente, e a área sensível à luz incidente torna-se maior. O potencial reverso aumenta a corrente escura (corrente no dispositivo na ausência de luz), uma vez que se torna mais provável que pares elétron-buraco gerados espontaneamente adquiram energia suficiente para que seus constituintes atinjam os terminais do dispositivo.

As características de um fotodiodo sem luz incidente são similares a de um diodo retificador comum. Quando o fotodiodo é polarizado diretamente, a corrente cresce exponencialmente com a tensão elétrica aplicada. Quando a polarização é reversa, uma pequena corrente de saturação aparece, e a corrente escura $I_{D}$ fica:

$$
I_{D}=I_{S A T}\left(e^{\frac{q V_{A}}{k_{B} T}}-1\right)
$$

onde $I_{S A T}$ é a corrente de saturação reversa do fotodiodo, $q$ é a carga elétrica, $T$ é a temperatura absoluta, $k_{B}$ é a constante de Boltzmann e $V_{A}$ é a voltagem aplicada na junção. Iluminando a junção com luz de frequência específica, a fotocorrente $I_{P}$ aparece no dispositivo e a corrente total $I_{T O T A L}$ fica:

$$
I_{T O T A L}=I_{S A T}\left(e^{\frac{q V_{A}}{k_{B} T}}-1\right)-\frac{Q e P}{h \nu}
$$

onde $Q$ é a eficiência quântica, $h$ é a constante de Planck, $\nu$ é a frequência do fóton incidente, $e$ é a carga elétrica e $P$ é o poder ótico incidente. O último termo da equação (2) é precisamente a expressão da fotocorrente $I_{P}$.

A corrente total cresce negativamente em função do aumento da intensidade luminosa sobre o fotodiodo (Fig. 2a). No equilíbrio termodinâmico, sem radiação incidente e sem polarização, a corrente líquida total, para um fotodiodo ideal, é nula (Fig. 2b).

Os detectores quânticos fazem a conversão direta dos fótons incidentes em um sinal elétrico, via transições eletrônicas em materiais semicondutores. Nesses materiais, a natureza estatística dos portadores de corrente dá origem ao ruído interno do dispositivo, mesmo na ausência de radiação incidente. Os dois processos fundamentais responsáveis por este ruído são as flutuações térmicas nas velocidades dos portadores livres e as flutuações na densidade de portadores, também devido às flutuações nas taxas de geração-recombinação térmicas.

Qualquer tipo de detector apresenta um limite mínimo de potência radiante detectável imposto por alguma forma de ruído, seja originária do próprio dispositivo, ou seja, da fonte de radiação incidente. Um detector atinge sua sensibilidade máxima, teoricamente admissível, quando limitado apenas pelo ruído quântico no 


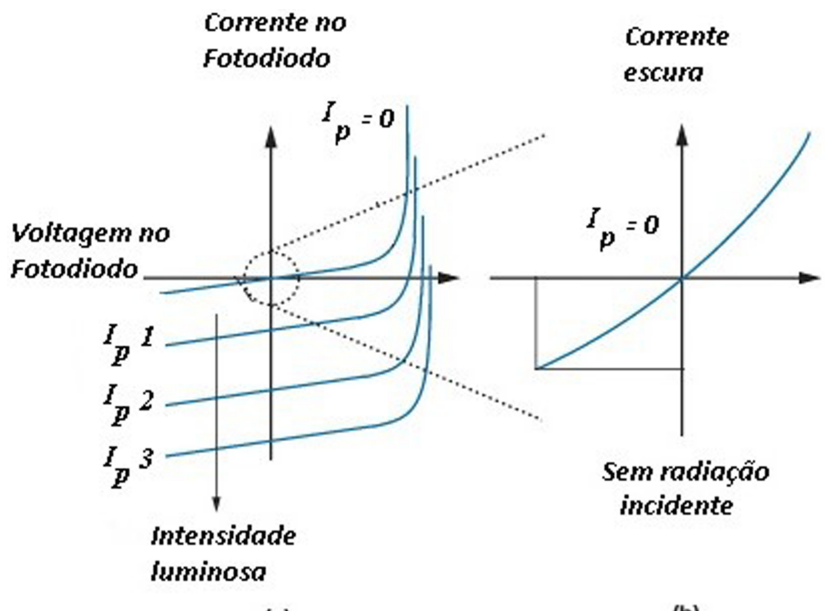

(a)

Figura 2: Função de transferência de um fotodiodo típico. Em (a) o andamento da curva de corrente em função da intensidade luminosa e em (b) o caso em que não existe radiação incidente na junção e se estabelece a corrente escura (dark current). Adaptado da Ref. [12].

sinal, isto é, aquele inerente às flutuações estatísticas na densidade de fótons emitidos pela própria fonte de sinal.

A fotocondutância tem sido muito útil tanto para investigações das propriedades físicas dos materiais como para aplicações tecnológicas em comunicações optoeletrônicas através de laser e sensores de luminosidade de alto desempenho. Os fotodetectores mais utilizados atualmente operam nas regiões visível e infravermelho próximo. Estes dispositivos não operam no infravermelho médio ou distante, pois os fótons com essa energia não são capazes de produzir pares elétron-lacuna.

\section{Projeto do sensor de luminosidade e testes de desempenho}

O medidor de luminosidade foi projetado tendo como base a plataforma Arduino e o módulo GY-302, que contém o sensor BH1750. A princípio, outros modelos de módulos disponíveis comercialmente, e que contém o sensor BH1750, podem ser utilizados para execução do projeto. O módulo contendo o sensor BH1750 incorpora os circuitos necessários para o seu funcionamento, incluindo um regulador de tensão e componentes passivos necessários. O sensor BH1750 é classificado como um sensor de luz ambiente com saída de dados digital. Internamente o sensor BH1750 inclui um fotodiodo de silício com resposta espectral na região do visível que se aproxima da resposta espectral do olho humano, um amplificador de sinais que converte a corrente elétrica gerada no fotodiodo em um sinal analógico de tensão, um conversor analógico-digital de 16 bits de resolução para digitalização da tensão gerada pelo amplificador, e um circuito digital lógico que calcula a iluminância medida em unidades de lux e efetua a comunicação de dados através do protocolo $\mathrm{I}^{2} \mathrm{C}$ [13]. Por ser um sensor integrado, a construção de um medidor de luminosidade com o BH1750 torna-se facilitada. O esquema de ligação do módulo contendo o sensor BH1750 e uma placa Arduino é mostrado na Fig. 3.

O módulo com o sensor BH1750 é alimentado com $+5 \mathrm{~V}$ disponível na placa Arduino utilizando os pinos VCC e GND. Para a comunicação digital através do protocolo $\mathrm{I}^{2} \mathrm{C}$ são utilizados os pinos A4 e A5 da placa Arduino, que são conectados nos pinos SLA (dados) e SLC (clock) do módulo com o sensor BH1750. Como é possível ter mais de um sensor BH1750 conectado no mesmo barramento $\mathrm{I}^{2} \mathrm{C}$, é necessário fixar o endereço do sensor BH1750 em 0x23 conectando o seu pino ADDR no terra.

O código computacional desenvolvido para a plataforma Arduino, e os detalhes para uso do sistema para aquisição de dados em tempo real no software Excel são apresentados no Apêndice deste trabalho. O software embarcado na placa Arduino configura o sensor BH1750 no modo de alta resolução com leitura contínua de dados, permitindo uma faixa de leitura de $1 \mathrm{~lx}$ até $65535 \mathrm{~lx}$ com resolução de $1 \mathrm{~lx}$.

Segundo o manual do fabricante do BH1750, no modo de alta resolução e leitura contínua o tempo de medida de iluminância é de até 0,2 s. Optou-se por efetuar as leituras de iluminância a cada $0,5 \mathrm{~s}$ aproximadamente, o que implica em uma taxa de 2 leituras por segundo. Podese modificar o código para elevar a taxa de leitura, porém deve-se levar em conta a limitação do tempo mínimo de $0,2 \mathrm{~s}$ entre as leituras.

Para certificar o funcionamento e exatidão das leituras foram realizadas medidas comparativas entre o medidor de luminosidade construído e o medidor comercial marca Instrutemp e modelo ITLD260. As medidas foram realizadas com o sensor de luminosidade BH1750 e o sensor de luminosidade do medidor comercial posicionados per-

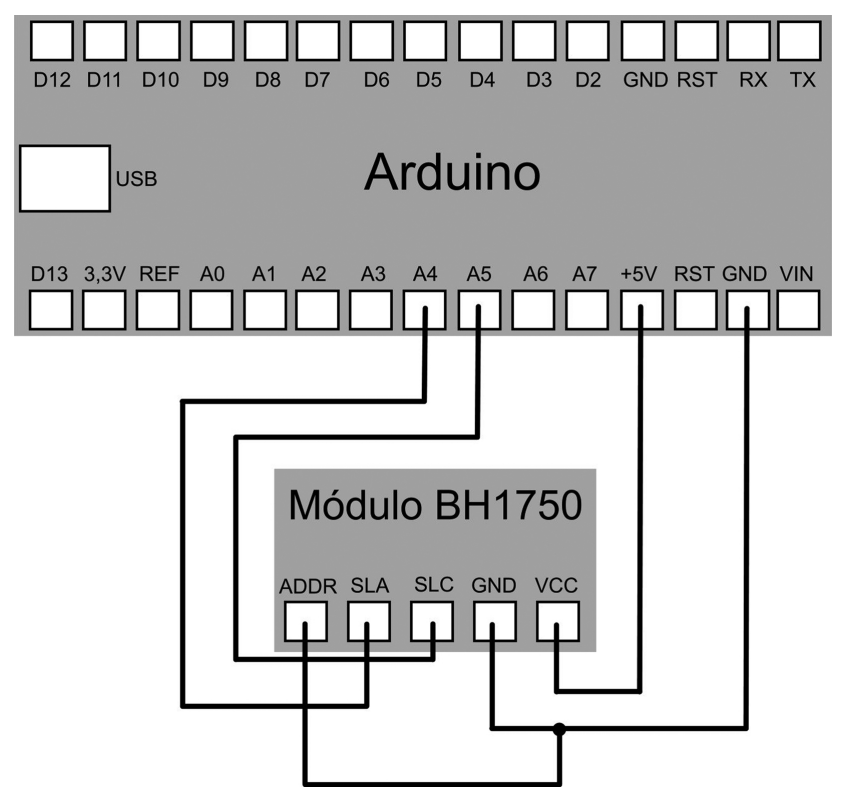

Figura 3: Esquema de montagem do medidor de luminosidade. 
pendicularmente à fonte luminosa. A distância da fonte luminosa e os sensores de luminosidade foi variada entre $5 \mathrm{~cm}$ e $30 \mathrm{~cm}$ para variação da intensidade luminosa sobre o sensor, mantendo os sensores fixos sobre uma mesa e alterando a altura da fonte luminosa. Foram realizadas leituras com uma luz fluorescente compacta de $11 \mathrm{~W}$ e uma lâmpada incandescente halógena de 18W/12V. Não foi possível efetuar as medidas simultâneas com o medidor comercial e com o sensor BH1750 pois é necessário manter cada um dos medidores exatamente em linha com a fonte luminosa e em uma posição fixa. A solução encontrada foi demarcar um gabarito sobre a mesa para a posição de cada medidor e trocá-los de posição para efetuar cada medida após a alteração da distância da fonte luminosa. Os resultados das medidas são mostrados na Fig. 4 (para a lâmpada incandescente) e na Fig. 5 (para a lâmpada fluorescente). Os dados foram submetidos a regressões lineares para ajuste na equação (3):

$$
\begin{aligned}
& \text { luminância (medidor comercial) }= \\
& \text { Iluminância (medidor construído) } a+b
\end{aligned}
$$

em que $a$ é o coeficiente angular, $b$ o coeficiente linear, e $R_{a d j}^{2}$ é o coeficiente de determinação ajustado.

As equações obtidas através de regressões lineares para as duas fontes luminosas resultaram em: $a=0,829 \pm 0,003$, $b=(-1 \pm 2)$ lx e $R_{a d j}^{2}=0,9998$ para a lâmpada incandescente, e $a=0,854 \pm 0,003, b=(3 \pm 2) \mathrm{lx}$ e $R_{a d j}^{2}=$ 0,9999 para a lâmpada fluorescente.

Para as duas fontes luminosas, os resultados indicam elevada correlação linear entre as medidas efetuadas com o medidor de luminosidade comercial e o medidor de luminosidade com o sensor BH1750. Os coeficientes angulares menores que 1,0 sugerem que as medidas efetuadas com o sensor BH1750 são subestimadas por um fator aproximadamente constante. Uma possível causa

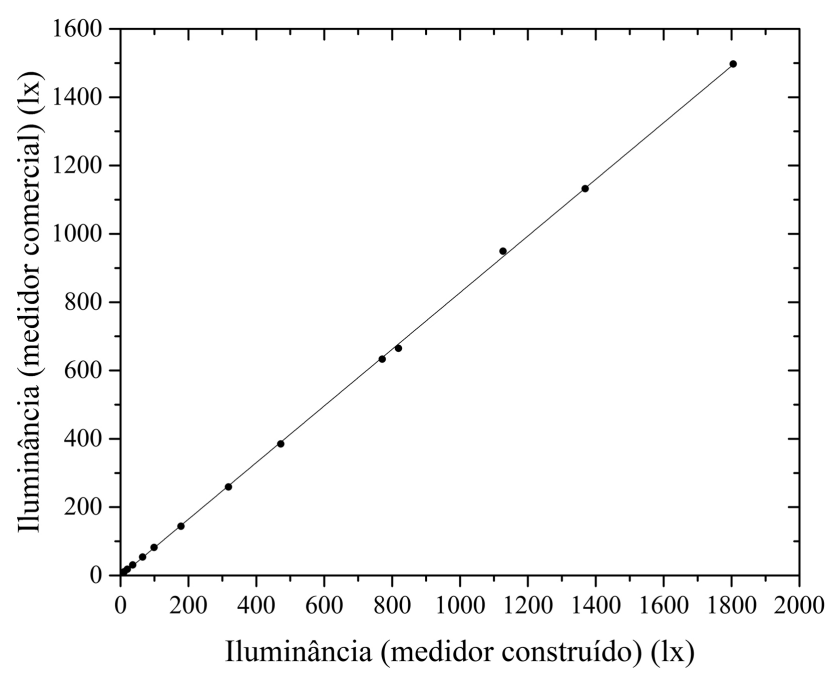

Figura 4: Curva de calibração do medidor de luminosidade utilizando uma lâmpada incandescente.

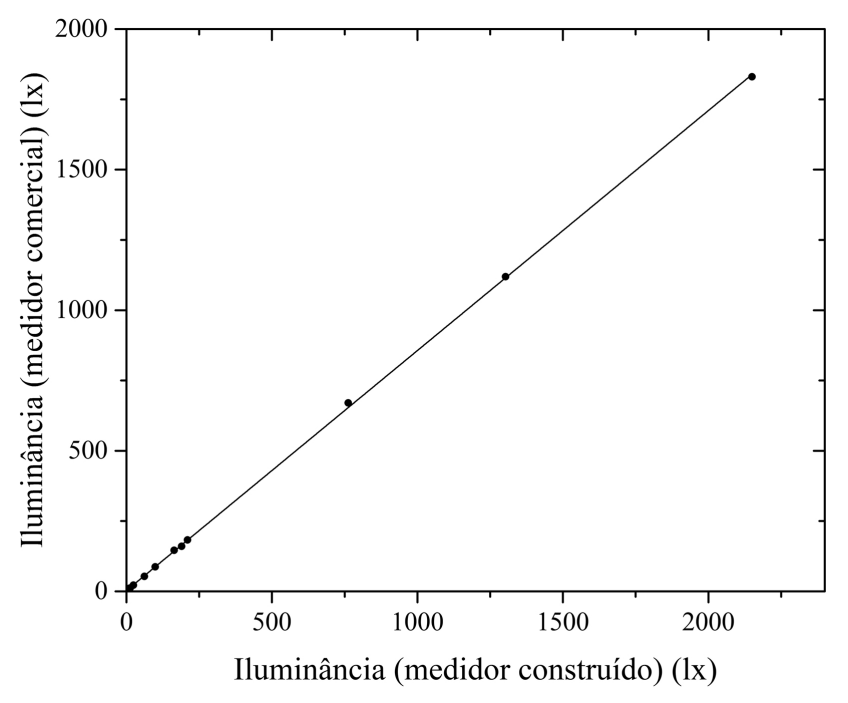

Figura 5: Curva de calibração do medidor de luminosidade utilizando uma lâmpada fluorescente compacta.

deste comportamento é a presença do difusor translúcido no sensor de luminosidade comercial, e que não foi utilizado no protótipo construído. Esse difusor tende a reduzir a iluminância incidente sobre o elemento sensor, e tem a função de tornar as medidas de iluminância menos dependentes do direcionamento da fonte luminosa. $\mathrm{O}$ valor médio dos coeficientes angulares determinados para as lâmpadas incandescente e fluorescente foi 0,842 . Este parâmetro foi utilizado para correção dos valores de iluminância medidos com o sensor BH1750. No código computacional apresentado na Fig. 12 do Apêndice, o fator de correção foi usado para calcular a iluminância segundo a equação (4):

$$
\begin{aligned}
& \text { Iluminância corrigida }= \\
& \text { Iluminância medida } \times 0,842
\end{aligned}
$$

A confiabilidade de protótipos construídos pode ser verificada utilizando o teste t emparelhado [14, p. 236], em que são utilizadas as medidas efetuadas com o sistema construído e com o sistema de referência (medidor comercial). O objetivo do teste $t$ emparelhado é verificar se há ou não diferença estatisticamente significativa entre as medidas efetuadas com os instrumentos construído e de referência. Utilizando os valores de iluminância corrigidos segundo a equação (4), e aplicando o teste $t$ emparelhado bilateral, com $95 \%$ de nível de confiança, foi determinado que não há diferenças significativas entre os valores de iluminância medidos com o medidor de luminosidade construído e com o medidor comercial. O valor-p calculado, para um conjunto de 27 medidas experimentais de iluminância, foi de 0,834 , indicando que há uma elevada probabilidade $(83,4 \%)$ de se rejeitar erroneamente a hipótese de que há diferenças significativas entre as medidas feitas com o instrumento construído e com o medidor comercial.

Os resultados dos testes de desempenho do sensor de luminosidade utilizando o sensor BH1750 mostram que 
a exatidão é suficiente para a execução de experimentos didáticos, em especial aqueles em que medidas relativas de iluminância são utilizadas.

\section{Aplicações do sensor de luminosidade}

Apresentamos a seguir três aplicações do sensor de luminosidade que podem ser utilizadas em atividades didáticas para o ensino de física e química. A primeira das aplicações utiliza o sensor de luminosidade para a investigação da variação da iluminância em função da distância da fonte de luz. A segunda aplicação utiliza o sensor de luminosidade como base para a construção do protótipo de um fotômetro de LED que pode ser aplicado em estudos de espectroscopia de absorção molecular. Na terceira aplicação foi enfatizado o uso da aquisição de dados em tempo real para o estudo da variação de iluminância de uma lâmpada fluorescente compacta durante o seu aquecimento interno.

\subsection{Atenuação da iluminância com a distância}

Uma aplicação do medidor de luminosidade no laboratório didático de física é a investigação da variação de iluminância com a distância da fonte de radiação luminosa. Aplicou-se o medidor de luminosidade com o sensor BH1750 em experimentos de levantamento de curvas de iluminância em função da distância, $d$, entre o sensor de luz e a fonte de radiação luminosa.

O modelo físico-matemático para a atenuação de uma fonte luminosa pontual e

isotrópica que emite com intensidade luminosa $l$, pode ser expresso de acordo com a equação (5):

$$
I=\frac{l}{d^{2}}
$$

em que $I$ é a iluminância no detector posicionado em linha com a fonte e a uma distância $d$.

Usando um sensor de luminosidade podemos estudar como a iluminância da fonte luminosa varia com a distância do detector e comparar esse resultado com o modelo matemático expresso pela equação 5 . Existem várias maneiras de medir o brilho relativo da luz, mas, independentemente da forma como a luz é medida, as mesmas variações relativas com a distância são observadas. Este exemplo de aplicação apresenta medidas da iluminância a uma variedade de distâncias de uma pequena fonte (lâmpada de LED de luz fria marca comercial Taschibra), para vermos como a iluminância varia com a distância. Em todos os casos, o plano de captação do sensor foi colocado nas diversas posições distantes do bulbo da lâmpada seguindo sempre perpendicular a uma linha reta de afastamento ortogonal ao centro do bulbo.

Obteve-se uma família de quatro curvas, para fontes luminosas de $5 \mathrm{~W}, 6 \mathrm{~W}, 7 \mathrm{~W}$ e $9 \mathrm{~W}$, mostrando o decaimento previsto pela equação 5 . Nestes experimentos, a distância entre o sensor de luz e a fonte de radiação luminosa foi variada de $80 \mathrm{~cm}$ até $180 \mathrm{~cm}$, mantendo-se o sensor em linha com a fonte luminosa. Importante lembrar que as lâmpadas não constituem verdadeiros pontos luminosos e sim fontes extensas de luz. O laboratório onde foi realizada a experiência foi escurecido tanto quanto possível e afastada toda e qualquer hipótese de superfícies refletoras próximas das lâmpadas para obter resultados satisfatórios. Deve-se captar apenas luminosidade primária da fonte, nunca via reflexões espúrias.

Pode-se linearizar a equação (5) traçando-se gráficos de iluminância em função de $1 / d^{2}$, conforme mostrado na Fig. 6. Efetuaram-se regressões lineares para os quatro conjuntos de dados e os resultados indicam elevadas correlações lineares, com coeficientes de correlação ajustados iguais a 0,999 para as curvas referentes às lâmpadas de $5 \mathrm{~W}, 6 \mathrm{~W}$ e $7 \mathrm{~W}$, e igual a 0,996 para a lâmpada de $8 \mathrm{~W}$. Tais resultados sugerem que o medidor de luminosidade respondeu conforme esperado.

Como extensão deste experimento, é possível aplicar a mesma metodologia e medir a iluminância expondo o sensor diretamente aos raios solares. Também é possível comparar este valor com os valores medidos pela exposição direta do sensor às lâmpadas a uma pequena distância destas (tipicamente alguns poucos centímetros). Uma proposta de atividade didática problematizadora pode consistir em: (a) Apontar o sensor de luz para o sol e medir a iluminância do sol em relação à lâmpada escolhida; (b) Questionar o aluno quantas lâmpadas ele teria que colocar a, por exemplo, $5 \mathrm{~cm}$ do sensor de luz para igualar a iluminância do sol?; (c) Usar o relacionamento matemático da equação 5 para calcular a iluminância se o sensor fosse hipoteticamente colocado à $5 \mathrm{~cm}$ do sol; (d) Determinar ainda quantas lâmpadas (escolha a potência) seriam equivalentes a esse valor. Ainda na linha de exploração do sensor, é possível aos alunos compararem as diversas tecnologias de fabricação de lâmpadas (filamento incandescente, fluorescente, gás, LED, etc...) e

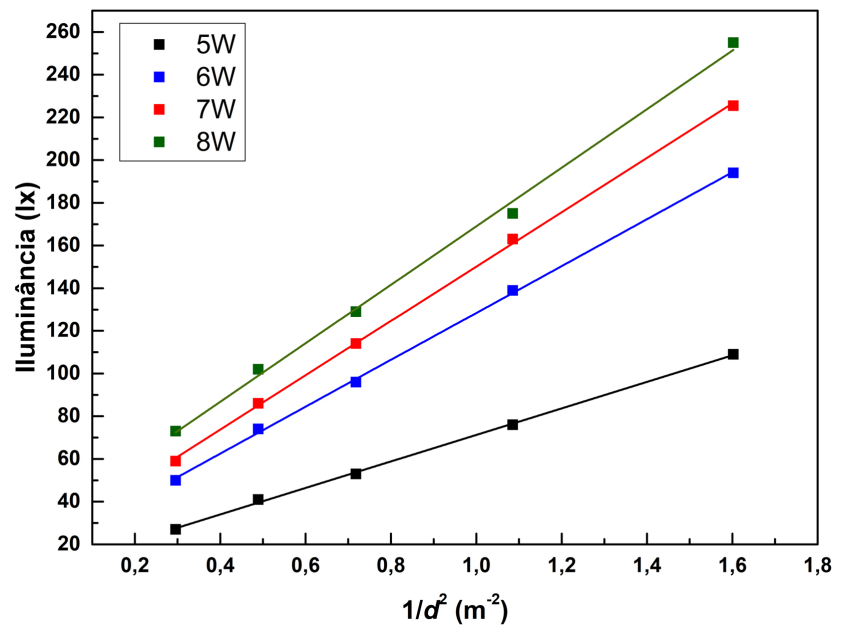

Figura 6: Curvas linearizadas de iluminância em função da distância de uma fonte de luz do sensor. 
também as formas geométricas oferecidas pelo mercado comercial.

\subsection{Fotômetro de LED}

Nesta aplicação, o medidor de luminosidade proposto foi utilizado como detector de radiação luminosa para construção de um fotômetro de LED simples. O fotômetro é um instrumento para medida da absorbância de uma amostra, e é utilizado em análises espectrofotométricas qualitativas e quantitativas. Trabalhos como os apresentados nas Ref. [15-16] apresentam propostas didáticas para fotômetros de LED construídos com materiais de baixo custo. Os detectores de luz mais comumente utilizados em trabalhos que descrevem a construção de fotômetros de LED são fotodiodos [17], fototransistores [3], fotoresistores (Light Dependent Resistors) [16] e LEDs [18]. No entanto, são raros na literatura trabalhos em que um sensor de luminosidade integrado tenha sido utilizado para compor um fotômetro de LED, podendo-se citar o trabalho de Anzalone, Glover e Pearce [19], que utilizam o sensor integrado Taos TSL230R. Considerase, portanto, relevante testar sua aplicabilidade de uso do sensor BH1750 para construção de um fotômetro de LED. Considera-se que a alternativa de utilizar o sensor BH1750 como detector de radiação apresenta a vantagem de maior simplicidade no projeto do fotômetro aliada a um projeto eletrônico interno mais refinado, sendo que o sensor BH1750 já incorpora internamente um fotodiodo, um circuito de amplificação de sinal e um digitalizador de alta resolução.

O fotômetro de LED proposto utiliza como fonte de radiação um LED que emite na região do visível, e apresenta um perfil de emissão aproximadamente monocromático. A radiação emitida pelo LED atravessa uma cubeta de vidro ou plástico transparente preenchida com o líquido a ser analisado, e a radiação não absorvida pela amostra emerge do lado oposto da cubeta e é medida pelo sensor de luminosidade BH1750. A absorbância, $A$, é definida de acordo com a equação (6), e pode ser interpretada como uma medida do grau de atenuação da radiação luminosa devido a uma amostra:

$$
A=-\log \left(\frac{I_{A}}{I_{B}}\right)
$$

em que $I_{A}$ é a intensidade de radiação luminosa que atravessa a amostra, e $I_{B}$ é a intensidade de radiação luminosa que chega no detector quando se utiliza o branco, que pode ser somente o solvente em que a amostra é solubilizada.

A Fig. 7 mostra um esquema da construção do fotômetro de LED, indicando a cubeta e o porta cubeta em corte transversal, e a disposição do LED e do sensor BH1750. A conexão do sensor BH1750 com a placa Arduino é feito utilizando o mesmo esquema da Fig. 3, e o software embarcado descrito no Apêndice, disponível no material suplementar.

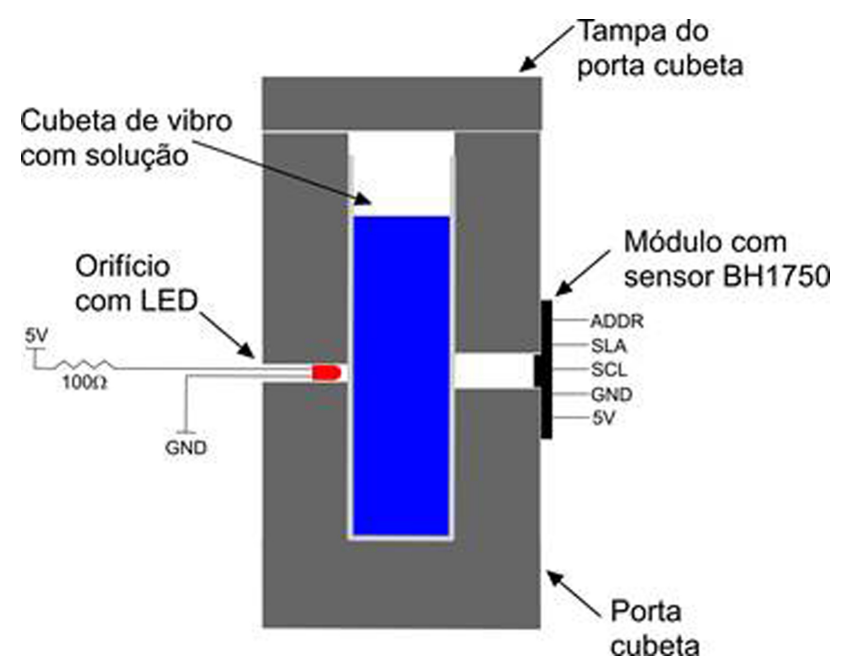

Figura 7: Esquema de construção do fotômetro de LED utilizando o sensor de luz $\mathrm{BH} 1750$.

A cubeta pode ser de plástico ou vidro, e o líquido deverá preencher ao menos $2 / 3$ de sua altura. Utilizou-se um LED vermelho de $5 \mathrm{~mm}$ de diâmetro com comprimento de onda de emissão em $620 \mathrm{~nm}$. A escolha do LED deve ser feita com base no comprimento de onda de máxima absorção da amostra a ser analisada. Nesta aplicação utilizamos como amostra soluções aquosas de $\mathrm{CuSO}_{4} .5 \mathrm{H}_{2} \mathrm{O}$, que absorvem fortemente próximo do comprimento de onda do LED vermelho. Para o acionamento do LED foi utilizada a saída de $5 \mathrm{~V}$ da placa Arduino com um resistor em série para limitação da corrente. $\mathrm{O}$ porta cubeta, esquematizado na Fig. 7, foi construído em plástico preto fosco e consiste em um poço para a cubeta com os orifícios de entrada e saída da radiação e uma tampa que tem como função minimizar possíveis interferências da luz ambiente durante as medidas. Uma foto do protótipo construído é mostrada na Fig. 8.

Para validar o funcionamento do fotômetro de LED foi determinada a curva para a Lei de Beer-Lambert de soluções aquosas de $\mathrm{CuSO}_{4} .5 \mathrm{H}_{2} \mathrm{O}$. A Lei de Beer-Lambert, apresentada na equação $(7)$, estabelece que a absorbância

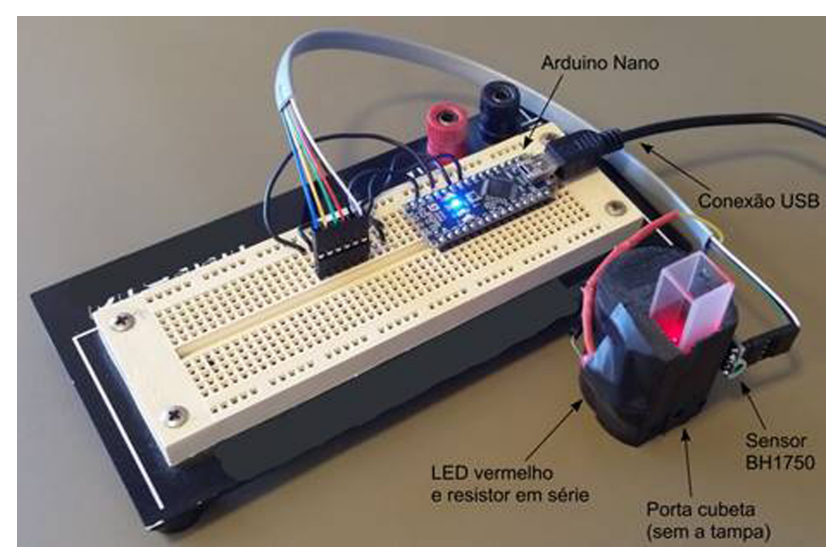

Figura 8: Foto do protótipo do fotômetro de LED. 
de uma amostra é proporcional à sua concentração molar $c$, à distância que a luz atravessa a amostra $l$, e a absortividade molar da amostra $\alpha$, que depende da identidade química da amostra e do comprimento de onda utilizado.

$$
A=\alpha l c
$$

Foram preparadas 9 soluções aquosas de $\mathrm{CuSO}_{4} .5 \mathrm{H}_{2} \mathrm{O}$, a partir do reagente $\mathrm{PA}$, com concentrações de 0,002 mol/L, 0,005 mol/L, 0,010 mol/L, 0,025 mol/L, 0,050 mol/L, 0,100 mol/L, 0,150 mol/L, 0,200 mol /L e 0,250 mol/L. O procedimento para medidas de absorbância consiste em (a) mede-se a iluminância no sensor de luminosidade quando a cubeta é preenchida somente com água destilada (branco); (b) substitui-se a água da cubeta pela solução a ser estudada e mede-se a iluminância no sensor de luminosidade; (c) calcula-se a absorbância de acordo com a equação (6).

A curva para a Lei de Lambert-Beer obtida, apresentada na Fig. 9, mostra uma correlação linear significativa, com coeficiente de correlação ajustado de 0,9992 obtido por regressão linear. Este resultado sugere que o sensor BH1750 apresenta resposta satisfatória e não foram observados desvios da Lei de Lambert-Beer na faixa de concentrações utilizada. Este trabalho limitou-se a apresentar potencialidades de uso do sensor BH1750 como sensor de luminosidade para construção de um fotômetro de LED, portanto não efetuamos a validação estatística da curva analítica apresentada na Fig. 9, o que pode ser feito em trabalhos posteriores. Com a curva analítica devidamente validada ela poderá ser utilizada para a determinação de concentrações desconhecidas a partir de medidas de absorbância. Alterando o tipo de LED, e, portanto, o comprimento de onda de sua emissão, é possível aplicar o fotômetro de LED para análises quantitativas de uma grande variedade de analitos. Deve-se

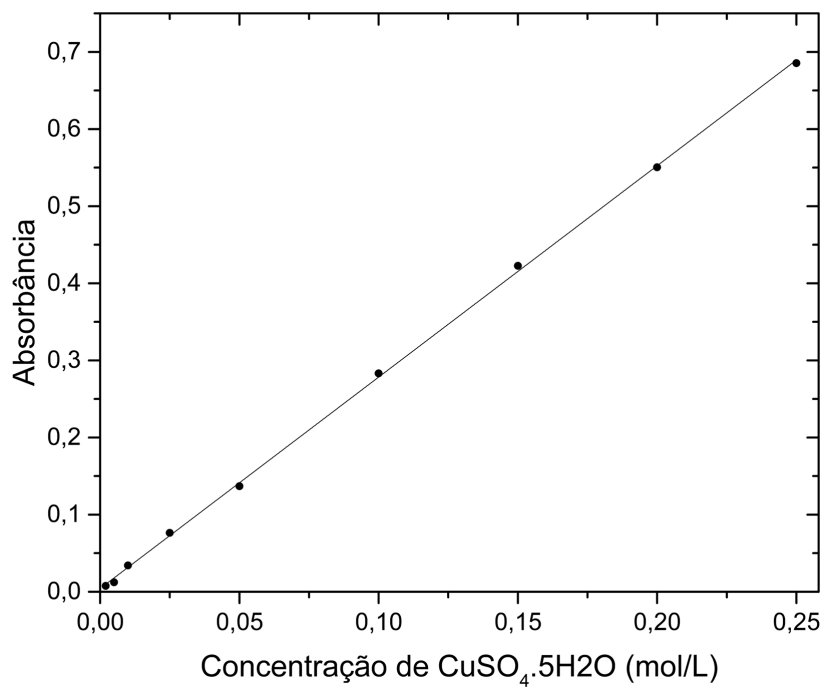

Figura 9: Curva experimental da Lei de Beer para uma solução de $\mathrm{CuSO}_{4} \cdot 5 \mathrm{H}_{2} \mathrm{O}$ determinada utilizando o fotômetro de LED construído. enfatizar algumas limitações quanto ao uso do sensor BH1750 como sensor de luz para a construção do fotômetro de LED. Uma das limitações se refere à velocidade de leitura relativamente baixa, o que pode limitar algumas aplicações em que o foco é a análise da cinética de reações químicas. Outra limitação é que a sensibilidade do sensor é baixa para radiação ultravioleta e infravermelho, restringindo sua aplicação em fotometria na região do visível.

\subsection{Aquisição de dados de iluminância em tempo real}

Em determinados experimentos, a aquisição de dados em tempo real permite fazer leituras frequentes que seriam inviáveis de serem efetuadas manualmente. Aplicou-se o medidor de luminosidade com sensor BH1750 para acompanhar a variação de iluminância de uma lâmpada fluorescente compacta após o seu acionamento. Foram efetuadas aproximadamente duas leituras por segundo por aproximadamente 4 minutos. A planilha e o gráfico adquirido em tempo real após a conclusão do experimento são mostrados na Fig. 10. A lâmpada fluorescente compacta encontrava-se inicialmente fria a uma temperatura de $18^{\circ} \mathrm{C}$, e foi mantida fixa a $10 \mathrm{~cm}$ de distância do sensor de luminosidade. Após cerca de $6 \mathrm{~s}$ do início da aquisição de dados, a lâmpada foi energizada e sua iluminância se elevou gradualmente até atingir seu valor máximo de aproximadamente $1200 \mathrm{~lx}$ após cerca de 3,2

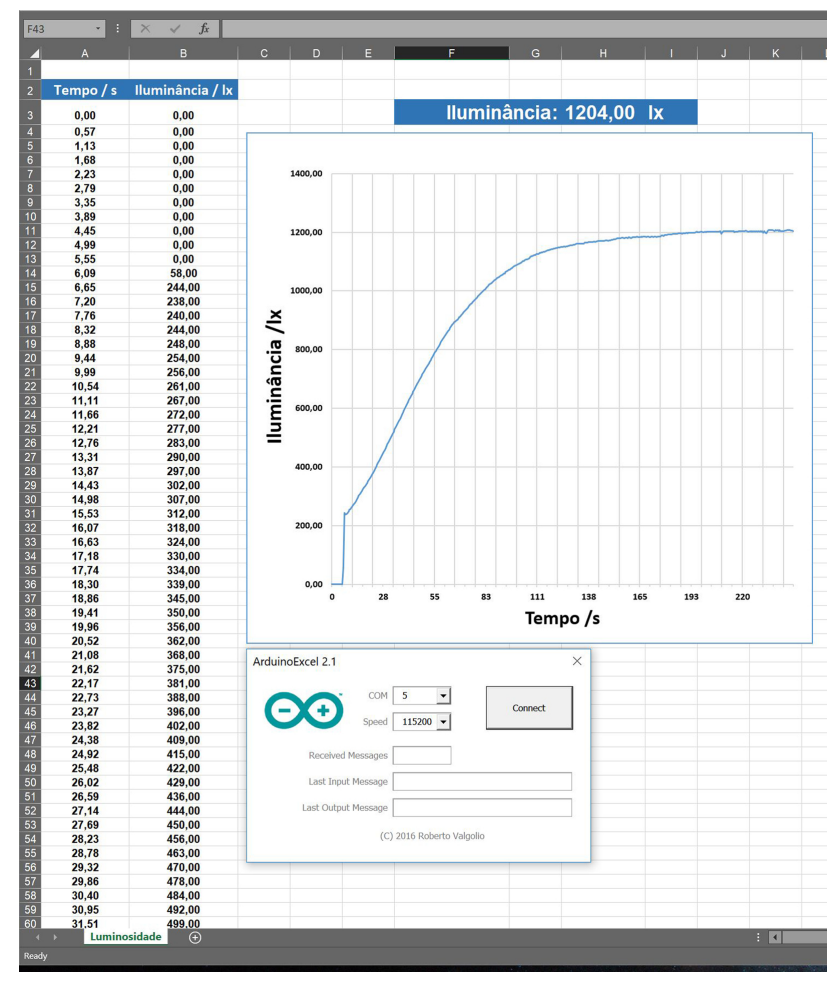

Figura 10: Captura de tela do software Excel mostrando a aquisição de dados de iluminância em tempo real para uma lâmpada fluorescente compacta após seu acionamento. 
min. A iluminância da lâmpada aumenta devido ao seu aquecimento interno após o seu acionamento. Se o mesmo experimento fosse efetuado iniciando de uma temperatura ambiente mais elevada possivelmente a lâmpada atingiria sua iluminância máxima mais rapidamente.

Nesta aplicação do sensor de luminosidade, a dinâmica da elevação da iluminância de uma lâmpada fluorescente compacta após o seu acionamento foi caracterizada. Outros experimentos que utilizam um arranjo experimental similar poderiam ser explorados, como por exemplo utilizar o medidor de luminosidade com o sensor BH1750, juntamente com um medidor de energia elétrica, para investigar a eficiência energética de lâmpadas de diferentes tecnologias.

\section{Considerações finais}

Apresentamos os detalhes de projeto de um medidor de luminosidade útil para ensino de ciências. Com a grande oferta de sensores e plataformas de desenvolvimento no mercado atual, e seu relativo baixo custo financeiro, se pode pensar num maior espaço para este dispositivo em atividades didáticas experimentais. As aplicações descritas neste trabalho mostram que é possível realizar um conjunto de experimentos didáticos interessantes, com um custo relativamente baixo e com relativa facilidade de construção. O sistema de medida descrito pode ser utilizado em atividades investigativas nas quais alunos e o professores utilizam sua criatividade para elaborar novas propostas de experimentos.

As práticas ilustrativas mostradas podem ser desenvolvidas em uma sala de aula comum com recursos de baixo custo. As características do sensor de luminosidade podem ser aproveitadas tanto com a finalidade de inovar e incrementar práticas de laboratório como para incentivar alunos a trabalhar em projetos interdisciplinares. Ao trabalhar em projetos similares, o estudante pode desenvolver conhecimentos sobre sensores eletrônicos, microcontroladores (e sua programação computacional) e os limites de validade da informação numérica experimental.

\section{Material Suplementar}

O seguinte Material suplementar está disponível on-line: Apêndice

\section{Referências}

[1] P.H. Guadagnini e V.E. Barlette, Revista Brasileira de Ensino Física 27, 369 (2005).

[2] P.H. Guadagnini, F.S. Rocha e V.E. Barlette, LatinAmerican Journal of Physics Education 5, 753 (2011).

[3] E. Lüdke, Revista Brasileira de Ensino de Física 32, 1506 (2010).

[4] F.S. Rocha e P.H. Guadagnini, Latin-American Journal of Physics Education 4, 306 (2009).
[5] T.A. Borges, Caderno Brasileiro de Ensino de Física 19, 291 (2002).

[6] R. Haag, I.S. Araujo e E.A. Veit, Física na Escola 6, 69 (2005).

[7] A.R. Hirdes, V.E. Barlette e P.H. Guadagnini, Vidya 38, 137 (2018).

[8] S.J. Metcalf e R.F. Tinker, Journal of Science Education and Technology 13, 43 (2004).

[9] D.R. Sokoloff, Latin-American Journal of Physics Education 6, 162012.

[10] D.R. Sokoloff e J. Benegas, in $2^{\circ}$ Taller Regional del Cono Sur sobre Aprendizaje Activo de la Física: Mecánica (AAMe, Córdoba, 2009).

[11] D.R. Sokoloff, P.W. Laws e R.K, Thornton. European Journal of Physics 28, 83 (2007).

[12] F. Jacob, Handbook of modern sensors: physics, designs, and applications (Spring-Verlag Inc., New York, 2004).

[13] Digital 16bit Serial Output Type Ambient Light Sensor IC BH1750FVI, disponível em: https://www.mouser. com/ds/2/348/bh1750fvi-e-186247.pdf, acessado em $20 / 09 / 2018$.

[14] J.I.D. Pinheiro, S.B. Cunha, S.R. Carvajal e G.C. Gomes, Estatística Básica: a arte de trabalhar com dados (Editora Elsevier, Rio de Janeiro, 2009).

[15] M.A.P. Leite, Fotômetro portátil de baixo custo: experimentação e contextualização no ensino de química da educação básica. Dissertação de Mestrado, Universidade Federal de Alagoas, Maceió (2014).

[16] P.C.C. Oliveira e M.A.P. Leite, Química Nova na Escola 38, 181 (2016).

[17] J.R. Hamilton, J.S. White e M.B. Nakhlen, Journal of Chemical Education 73, 1052 (1996).

[18] E.V. Kvittingen, L. Kvittingen, B.J. Sjursnes e R. Verley, Journal of Chemical Education 93, 1814 (2016).

[19] G.C. Anzalone, A.G. Glover e J.M. Pearce, Sensors 13, 5338 (2013). 